\title{
Article
}

\section{Existence and uniqueness for delay fractional differential equations with mixed fractional derivatives}

\author{
Ahmed Hallaci ${ }^{1}$, Hamid Boulares ${ }^{1}$ and Abdelouaheb Ardjouni ${ }^{2,3, *}$ \\ 1 Department of Mathematics, Faculty of Sciences, University of 08 Mai 1945 Guelma, P. Box 401, Guelma, 24000, \\ Algeria. \\ 2 Faculty of Sciences and Technology, Department of Mathematics and Informatics, Univ Souk Ahras, P.O. Box 1553, \\ Souk Ahras, 41000, Algeria. \\ 3 Applied Mathematics Lab, Faculty of Sciences, Department of Mathematics, Univ Annaba, P.O. Box 12, Annaba \\ 23000, Algeria. \\ * Correspondence: abd_ardjouni@yahoo.fr
}

Received: 18 April 2020; Accepted: 17 June 2020; Published: 13 August 2020.

\begin{abstract}
Using the Krasnoselskii's fixed point theorem and the contraction mapping principle we give sufficient conditions for the existence and uniqueness of solutions for initial value problems for delay fractional differential equations with the mixed Riemann-Liouville and Caputo fractional derivatives. At the end, an example is given to illustrate our main results.
\end{abstract}

Keywords: Mixed derivatives, delay fractional differential equations, fixed point theorems.

MSC: 34A08, 34K05.

\section{Introduction}

$\mathbf{T}$ he fractional differential equations is a hot topic of research due to its various applications in many scientific disciplines such as physics, chemistry, biology, engineering, viscoelasticity, signal processing, electrotechnical, electrochemistry and controllability, see [1-6] and the references therein. The neutral fractional differential equations have been studied extensively in the last decades and different technics have been used to solve it, for example, fixed point theorems, upper and lower solution method, spectral theory, etc. For some recent contributions in fractional boundary value problems, we refer [7-9] and the references therein. To the best of our knowledge, the use of mixed fractional derivative in neutral fractional differential equations which is an important type of fractional differential equations is still not sufficiently generalized. Our main aim is to solve mixed fractional differential equations.

Benchohra et al. [7], investigated the existence of solutions for the following Riemann-Liouville fractional order functional differential equation with infinite delay

$$
\begin{cases}{ }^{R L} D^{\alpha}\left[u(t)-g\left(t, u_{t}\right)\right]=f\left(t, u_{t}\right), & t \in[0, T], 0<\alpha<1, \\ u(t)=\phi(t), & t \in(-\infty, 0] .\end{cases}
$$

Agarwal et al. [8], studied the initial value problem of fractional neutral Caputo fractional derivative

$$
\left\{\begin{array}{l}
{ }^{C} D^{\alpha}\left[u(t)-g\left(t, u_{t}\right)\right]=f\left(t, u_{t}\right), t \in\left(t_{0}, \infty\right), t_{0} \geq 0,0<\alpha<1, \\
u_{t_{0}}=\phi
\end{array}\right.
$$

and established the existence results of solutions of this problem by using Krasnoselskii's fixed point theorem. In [9], Ahmad et al. studied the existence and uniqueness of solutions to the following boundary value problem 


$$
\begin{cases}D^{\alpha}\left(D^{\beta} u(t)-g\left(t, u_{t}\right)\right)=f\left(t, u_{t}\right), & t \in[1, b], \\ u(t)=\phi(t), & t \in[1-\tau, 1], \\ D^{\beta} u(1)=\eta \in \mathbb{R}, & \end{cases}
$$

where $D^{\alpha}$ and $D^{\beta}$ are the Caputo-Hadamard fractional derivatives, $0<\alpha, \beta<1$.

Motivated and inspired by above mentioned works, in this paper we investigate the existence and uniqueness of solutions for the following initial value problem of the mixed Riemann-Liouville and Caputo fractional functional differential equation with delay

$$
\begin{cases}{ }^{R L} D^{\alpha}\left[{ }^{C} D^{\beta} u(t)-g(t, u(t-\tau))\right]=f(t, u(t-\tau)), & t \in I=[0, T], \\ u(t)=\phi(t), & t \in[-\tau, 0], \\ \lim _{t \rightarrow 0} t^{1-\alpha}{ }^{C} D^{\beta} u(t)=0, & u^{\prime}(0)=0,\end{cases}
$$

where ${ }^{R L} D^{\alpha}$ and ${ }^{C} D^{\beta}$ are the Riemann Liouville and the Caputo fractional derivatives respectively, $0<\alpha<1$, $1<\beta<2, f, g \in I \times \mathbb{R} \rightarrow \mathbb{R}$ are given continuous functions and $\phi \in C([-\tau, 0], \mathbb{R})$. To show the existence of solutions, we transform (1) into an integral equation and then use Krasnoselskii's fixed point theorem. The obtained integral equation splits in the sum of two mappings, one is a contraction and the other is compact. Also, by utilizing the contraction mapping principle, we give uniqueness results.

The organization of this paper is as follows: In Section 2 we recall some useful preliminaries and present the equivalent fixed point problem corresponding to (1). In Section 3, we discuss the existence and uniqueness of solutions for (1) via fixed point theory. An example is constructed for illustrating the obtained results.

\section{Preliminaries}

In this section, we introduce notation, definitions, and preliminary facts that we need in the sequel. By $C(I, \mathbb{R})$ we mean the Banach space of all continuous functions from $I$ into $\mathbb{R}$ with the norm

$$
\|u\|_{\infty}:=\sup \{|u(t)|: t \in I\} .
$$

Also $C_{\tau}=C([-\tau, 0], \mathbb{R})$ is endowed with norm

$$
\|\phi\|_{C}:=\sup \{|\phi(t)|: t \in[-\tau, 0]\} .
$$

Definition $1([2,4,6])$. The Riemann-Liouville fractional integral of the function $u$ of order $\alpha>0$ is defined by

$$
I^{\alpha} u(t)=\frac{1}{\Gamma(\alpha)} \int_{0}^{t} \frac{u(s)}{(t-s)^{1-\alpha}} d s,
$$

where $\Gamma$ is the Euler gamma function defined by $\Gamma(\alpha)=\int_{0}^{\infty} e^{-t} t^{\alpha-1} d t$.

Definition 2 ([2,4,6]). The Riemann-Liouville fractional derivative of the function $u$ of order $\alpha \in(n-1, n]$ is defined by

$$
{ }^{R L} D^{\alpha} u(t)=\frac{1}{\Gamma(n-\alpha)} \frac{d^{n}}{d t^{n}} \int_{0}^{t} \frac{u(s)}{(t-s)^{\alpha-n+1}} d s .
$$

Definition $3([2,4,6])$. The Caputo fractional derivative of the function $u$ of order $\alpha \in(n-1, n]$ is defined by

$$
{ }^{C} D^{\alpha} u(t)=\frac{1}{\Gamma(n-\alpha)} \int_{0}^{t} \frac{u^{(n)}(s)}{(t-s)^{\alpha-n+1}} d s .
$$

Let $\alpha>0$ be a real number, we have following results: 
Lemma 1 ([4]). The unique solution of the linear fractional differential equation

$$
{ }^{R L} D^{\alpha} u(t)=0,
$$

is given by

$$
u(t)=c_{1} t^{\alpha-1}+c_{2} t^{\alpha-2}+c_{3} t^{\alpha-3}+\ldots+c_{n} t^{\alpha-n}, c_{i} \in \mathbb{R}, i=1,2, \ldots, n .
$$

where $n=[\alpha]+1,[\alpha]$ denotes the integer part of $\alpha$.

Lemma 2 ([4]). The unique solution of the linear fractional differential equation

$$
{ }^{C} D^{\alpha} u(t)=0,
$$

is given by

$$
u(t)=c_{1}+c_{2} t+\ldots+c_{n} t^{n-1}, c_{i} \in \mathbb{R}, i=1,2, \ldots, n,
$$

where $n=[\alpha]+1$.

Lemma 3. (1) is equivalent to the following integral equation

$$
\begin{aligned}
u(t) & =\frac{1}{\Gamma(\alpha+\beta)} \int_{0}^{t}(t-s)^{\alpha+\beta-1} f(s, u(s-\tau)) d s \\
& +\frac{1}{\Gamma(\beta)} \int_{0}^{t}(t-s)^{\beta-1} g(s, u(s-\tau)) d s+\phi(0) .
\end{aligned}
$$

Proof. Using Lemma 1, equation one of (1) can be written as

$$
{ }^{C} D^{\beta} u(t)=I^{\alpha} f(t, u(t-\tau))+g(t, u(t-\tau))+c_{0} t^{\alpha-1} .
$$

Using the condition $\lim _{t \rightarrow 0} t^{1-\alpha}{ }^{C} D^{\beta} u(t)=0$, we get $c_{0}=0$. On the other hand, from Lemma 2 , one gets

$$
u(t)=I^{\alpha+\beta} f(t, u(t-\tau))+I^{\beta} g(t, u(t-\tau))+c_{1}+c_{2} t .
$$

Clearly $u(0)=\phi(0)$, so we obtain $c_{1}=\phi(0)$ and because $u^{\prime}(0)=0$, we find $c_{2}=0$, then we get the integral equation

$$
\begin{aligned}
u(t)= & \frac{1}{\Gamma(\alpha+\beta)} \int_{0}^{t}(t-s)^{\alpha+\beta-1} f(s, u(s-\tau)) d s \\
& +\frac{1}{\Gamma(\beta)} \int_{0}^{t}(t-s)^{\beta-1} g(s, u(s-\tau)) d s+\phi(0) .
\end{aligned}
$$

Our main results are based on the following Krasnoselskii fixed point theorem and the contraction mapping principle.

Theorem 1 (Krasnoselskii fixed point theorem [10,11]). If $\mathcal{M}$ is a nonempty bounded, closed and convex subset of $a$ Banach space $E$ and $\mathcal{A}$ and $\mathcal{B}$ two operators defined on $\mathcal{M}$ with values in $E$ such that

i) $\mathcal{A} x+\mathcal{B} y \in \mathcal{M}$, for all $x, y \in \mathcal{M}$,

ii) $\mathcal{A}$ is continuous and compact,

iii) $\mathcal{B}$ is a contraction.

Then there exists $z \in \mathcal{M}$ such that $z=\mathcal{A} z+\mathcal{B} z$.

Theorem 2 (Contraction mapping principle [10,11]). Let $E$ be a Banach space. If $\mathcal{H}: E \rightarrow E$ is a contraction, then $\mathcal{H}$ has a unique fixed point in $E$. 


\section{Main results}

We express (2) as

$$
u(t)=(\mathcal{A} u)(t)+(\mathcal{B} u)(t)=(\mathcal{H} u)(t),
$$

where the operators $\mathcal{A}, \mathcal{B}: C([-\tau, T], \mathbb{R}) \rightarrow C([-\tau, T], \mathbb{R})$ are defined by

$$
\begin{gathered}
(\mathcal{A} u)(t)= \begin{cases}0, & \text { if } t \in[-\tau, 0], \\
\frac{1}{\Gamma(\alpha+\beta)} \int_{0}^{t}(t-s)^{\alpha+\beta-1} f(s, u(s-\tau)) d s & \text { if } t \in I,\end{cases} \\
(\mathcal{B} u)(t)= \begin{cases}\phi(t), & \text { if } t \in[-\tau, 0], \\
\frac{1}{\Gamma(\beta)} \int_{0}^{t}(t-s)^{\beta-1} g(s, u(s-\tau)) d s+\phi(0) & \text { if } t \in I .\end{cases}
\end{gathered}
$$

Consider the following hypothesis:

(H1) $f, g: I \times \mathbb{R} \rightarrow \mathbb{R}$ are continuous functions.

(H2) There exists $\eta \in C\left(I, \mathbb{R}_{+}^{*}\right)$ such that

$$
|g(t, u)-g(t, v)| \leq \eta(t)\|u-v\|_{[-\tau, T]}, g(t, 0)=0 .
$$

(H3) There exists $\zeta \in C\left(I, \mathbb{R}_{+}^{*}\right)$ such that

$$
|f(t, u)| \leq \zeta(t)
$$

Theorem 3. Assume that (H1) - (H3) hold. Then (1) has at least one solution on $[-\tau, T]$, provided

$$
\frac{T^{\beta}\|\eta\|_{\infty}}{\Gamma(\beta+1)}<1
$$

Proof. We show that the operators $\mathcal{A}$ and $\mathcal{B}$ defined by (4) and (5) satisfied all hypothesis of Theorem 1. Choosing $R \geq \frac{\|\phi\|_{C}+\frac{T^{\alpha+\beta}}{\Gamma(\alpha+\beta+1)}\|\zeta\|_{\infty}}{1-\frac{T^{\beta}\|\eta\|_{\infty}}{\Gamma(\beta+1)}}$ and define $B_{R}=\left\{u \in C([-\tau, T], \mathbb{R}):\|u\|_{[-\tau, T]} \leq R\right\}$, then for any $u, v \in B_{R}$, we have

$$
\begin{aligned}
|(\mathcal{A} u)(t)+(\mathcal{B} v)(t)| & \leq \frac{1}{\Gamma(\alpha+\beta)} \int_{0}^{t}(t-s)^{\alpha+\beta-1} f(s, u(s-\tau)) d s+|\phi(0)|+\frac{1}{\Gamma(\beta)} \int_{0}^{t}(t-s)^{\beta-1} g(s, u(s-\tau)) d s \\
& \leq\|\phi\|_{C}+\frac{T^{\alpha+\beta}}{\Gamma(\alpha+\beta+1)}\|\zeta\|_{\infty}+\frac{T^{\beta} R}{\Gamma(\beta+1)}\|\eta\|_{\infty} \leq R
\end{aligned}
$$

This means that $\mathcal{A} u+\mathcal{B} v \in B_{R}$ for any $u, v \in B_{R}$. On the other hand, operator $\mathcal{A}$ is continuous from the continuity of $f$.

It remains to prove the compactness of the operator $\mathcal{A}$. Clearly, (7) show that $\mathcal{A}$ is uniformly bounded on $B_{R}$ and

$$
\|\mathcal{A} v\| \leq \frac{T^{\alpha+\beta}}{\Gamma(\alpha+\beta+1)}\|\zeta\|_{\infty}
$$

Also, for $t_{1}, t_{2} \in[0, T], t_{1}<t_{2}$ we have

$$
\begin{aligned}
\left|(\mathcal{A} u)\left(t_{2}\right)-(\mathcal{A} u)\left(t_{1}\right)\right| & \frac{1}{\Gamma(\alpha+\beta)} \int_{0}^{t_{1}}\left[\left(t_{2}-s\right)^{\alpha+\beta-1}-\left(t_{1}-s\right)^{\alpha+\beta-1}\right]|f(s, u(s-\tau))| d s \\
& +\frac{1}{\Gamma(\alpha+\beta)} \int_{t_{1}}^{t_{2}}\left(t_{2}-s\right)^{\alpha+\beta-1}|f(s, u(s-\tau))| d s \\
& =\frac{\|\zeta\|_{\infty}}{\Gamma(\alpha+\beta+1)}\left(t_{2}^{\alpha+\beta}-t_{1}^{\alpha+\beta}\right),
\end{aligned}
$$

which is independent of $u$ and tends to zero as $t_{2} \rightarrow t_{1}$. Thus, the set $\left\{\mathcal{A} u, u \in B_{R}\right\}$ is equicontinuous and hence it is relatively compact. So, by Ascoli-Arzela theorem, $\mathcal{A}$ is compact on $B_{R}$. 
It remains to show that $\mathcal{B}$ is a contraction. Let $u, v \in C([-\tau, T], \mathbb{R})$, then for all $t \in[-\tau, T]$, we have

$$
\begin{aligned}
|(\mathcal{B} u)(t)-(\mathcal{B} v)(t)| & \leq \frac{1}{\Gamma(\beta)} \int_{0}^{t}(t-s)^{\beta-1}|g(s, u(s-\tau))-g(s, v(s-\tau))| d s \\
& \leq \frac{T^{\beta}\|\eta\|_{\infty}}{\Gamma(\beta+1)}\|u-v\|_{[-\tau, T]}
\end{aligned}
$$

from (6) $\mathcal{A}$ is a contraction operator.

Thus all the assumptions of Theorem 1 are satisfied. So the conclusion of Theorem 3 implies that (1) has at least one continuous solution on $[-\tau, T]$.

Now, we use the contraction principle mapping to investigate uniqueness results for (1).

Theorem 4. Assume that (H1) - (H3) hold. Then (1) has a unique solution on $[-\tau, T]$, provided

$$
\frac{T^{\alpha+\beta}\|\zeta\|_{\infty}}{\Gamma(\alpha+\beta+1)}+\frac{T^{\beta}\|\eta\|_{\infty}}{\Gamma(\beta+1)}<1
$$

Proof. We claim that $\mathcal{H}$ is contraction mapping, this show that $\mathcal{H}$ has a unique fixed point which is the unique solution of (1). To this end, let $u, v \in C([-\tau, T], \mathbb{R})$, then for all $t \in[-\tau, T]$, we have

$$
\begin{aligned}
|(\mathcal{H} u)(t)-(\mathcal{H} v)(t)| \leq & \frac{1}{\Gamma(\alpha+\beta)} \int_{0}^{t}(t-s)^{\alpha+\beta-1}|f(s, u(s-\tau))-f(s, v(s-\tau))| d s \\
& +\frac{1}{\Gamma(\beta)} \int_{0}^{t}(t-s)^{\beta-1}|g(s, u(s-\tau))-g(s, v(s-\tau))| d s \\
& \leq\left[\frac{T^{\alpha+\beta}\|\zeta\|_{\infty}}{\Gamma(\alpha+\beta+1)}+\frac{T^{\beta}\|\eta\|_{\infty}}{\Gamma(\beta+1)}\right]\|u-v\|_{[-\tau, T]} .
\end{aligned}
$$

Therefore $\mathcal{H}$ is a contraction. Thus, the conclusion of Theorem 7 follows by the contraction mapping principle.

Now, we give an example to illustrate the usefulness of our main results.

Example 1. Consider (1) with $\alpha=0.5, \beta=1.5, T=1, f(t, x)=\frac{\sin \left(t^{2} \arctan x^{3}\right)}{1+t^{2}}, g(t, x)=$ $\cos t\left(t^{2} e^{-e^{-x}}-\frac{t^{2}}{e}+\frac{\sin t x}{e}\right)$. Clearly $f$ and $g$ are continuous functions and

$$
|f(t, x)| \leq \frac{\pi}{2} \frac{t^{2}}{1+t^{2}}=\zeta(t)
$$

and

$$
g(t, 0)=0,|g(t, x)-g(t, y)| \leq \frac{t^{2}}{e} \cos t\|x-y\|_{[-\tau, T]}=\eta(t)\|x-y\|_{[-\tau, T]},
$$

(since $z+e^{-z} \geq 1$ for all real $z$ then one gets $\left|e^{-e^{-x}}-e^{-e^{-y}}\right| \leq \frac{|x-y|}{e}$ ). Also, $\frac{T^{\beta}\|\eta\|_{\infty}}{\Gamma(\beta+1)} \simeq 0.27674<1$. Thus, by Theorem 3, (1) with above data has at least one solution on $[-\tau, 1]$.

Author Contributions: All authors contributed equally to the writing of this paper. All authors read and approved the final manuscript.

Conflicts of Interest: "The authors declare no conflict of interest."

\section{References}

[1] Das, S. (2011). Functional fractional calculus. Springer Science \& Business Media.

[2] Hilfer, R. (Ed.). (2000). Applications of fractional calculus in physics (Vol. 35, No. 12, pp. 87-130). Singapore: World scientific.

[3] Khare, A. (2005). Fractional statistics and quantum theory. World Scientific. 
[4] Kilbas, A. A., Srivastava, H. M., \& Trujillo, J. J. (2006). Theory and applications of fractional differential equations (Vol. 204). Elsevier Science B. V., Amsterdam.

[5] Petráš, I. (2011). Fractional-order nonlinear systems: modeling, analysis and simulation. Springer Science \& Business Media.

[6] Podlubny, I. (1998). Fractional differential equations: an introduction to fractional derivatives, fractional differential equations, to methods of their solution and some of their applications. Elsevier.

[7] Benchohra, M., Henderson, J., Ntouyas, S. K., \& Ouahab, A. (2008). Existence results for fractional order functional differential equations with infinite delay. Journal of Mathematical Analysis and Applications, 338(2), 1340-1350.

[8] Agarwal, R. P., Zhou, Y., \& He, Y. (2010). Existence of fractional neutral functional differential equations. Computers $\mathcal{E}$ Mathematics with Applications, 59(3), 1095-1100.

[9] Ahmad, B., \& Ntouyas, S. K. (2017). Existence and uniqueness of solutions for Caputo-Hadamard sequential fractional order neutral functional differential equations. Electronic Journal of Differential Equations, 2017(36), 1-11.

[10] Smart, D. R. (1980). Fixed point theorems (Vol. 66). Press, Cambridge.

[11] Zeidler, E. (1985). Nonlinear analysis and its applications I: Fixed Point theorems. Springer-Verlag New York, Inc

(C) 2020 by the authors; licensee PSRP, Lahore, Pakistan. This article is an open access article distributed under the terms and conditions of the Creative Commons Attribution (CC-BY) license (http://creativecommons.org/licenses/by/4.0/). 